\title{
Small colony variant of Escherichia coli isolated from a recurrent urinary tract infection- a case report
}

\author{
Shivendra Dutt ${ }^{1 *}$; Nagalakshmi ${ }^{2}$; Shobha K. L ${ }^{2}$ \\ ${ }^{I}$ Department of Microbiology, Kasturba Medical College, Manipal, Karnataka 576104, India \\ ${ }^{2}$ Department of Microbiology, Melaka Manipal Medical College (Manipal Campus) Manipal University, \\ Manipal, Karnataka 576104, India \\ *Corresponding author E-mail: shobha.kl@manipal.edu
}

\begin{abstract}
Introduction: Small colony variant of Escherichia coli may cause persistent and recurrent infections in human. These slow growing phenotypic variants are difficult to identify and mislead the diagnosis which can lead to choice of inappropriate antibiotics. In this case report we are presenting a case of recurrent urinary tract infection which was associated with small colony variants of E. coli.

Case presentation: A two year old male child was admitted to the hospital with a complaint of passing thin stream of urine. One month earlier the child had been previously diagnosed with bilateral grade five Vesicoureteral reflux. Child had developed recurrent urinary tract infection caused by small colony variants of E. coli.

Conclusion: child had undergone bilateral ureter reimplantation as part of treatment to Vesicoureteral reflux. Since vesicoureteral reflex condition is one of the risk factor to develop urinary tract infections. Repeated exposure to antibiotic might have been induced stress condition to bacteria and emerged as slow growing sub population of E. coli.
\end{abstract}

Keywords: Escherichia coli, Small Colony Variant, Urinary Tract Infections.

\section{Introduction}

Urinary tract infections (UTI) are a common form of infection in infants and children. Diagnosis of UTI in young children and infants is very important as early detection plays a critical role for the preservation of renal function of the growing kidney (Habib S, 2012). Microbiological investigation of urine specimen is necessary for the identification of pathogens and antibiotic susceptibility (Hooton TM \& Stamm WE, 1997). The most common pathogen associated with UTI is the uropathogenic Escherichia coli (UPEC), however some individuals have an increased predisposition to recurrences of UTI due to the initially infecting strain which even after elimination from urinary tract may persist in the faecal flora. Few studies reported that Escherichia coli can invade bladder epithelial cells and can form large bacterial inclusions after replication intracellularly (Foxman B, 1990 \& Mulvey MA et al., 1998). Small colony variants (SCVs) are known as slow growing subpopulations that form small, non-pigmented and nonhemolytic colonies. Deficiencies in electron transport activities lead to auxotrophism for thymidine, menadione, or hemin causing the typical biochemical characteristics of the variants (Proctor RA et al., 2006 \& L J Mandel et al., 1984). Studies show that 3-5 \% of persistent infections may be caused by these small colony Variant Escherichia coli (Charlotte A. C. et al., 1946 \& Roggenkamp A et al., 1998). Clinically these small colony variants are better able to persist in mammalian cells and are less susceptible to antibiotics (Harald S et al., 2003 \& Proctor RA et al., 1995). Due to their unusual phenotypical and biochemical characteristics, they pose a challenge for clinical microbiologists. This problem might lead to diagnostic underestimation, which will cause therapeutic failures in the clinical settings (Vaudaux $P$ et al., 2006). Small colony variant Escherichia coli are reported to cause persistent and recurrent infections with more chances of treatment failure than those caused by wild type. However, there is limited literature regarding this. Here, we describe the case of a recurrent UTI infections caused by Small colony variant Escherichia coli, which occurred after undergoing surgery for bilateral grade five vesicouteral reflexes.

\section{Case presentation}

A two year old male child was admitted to the hospital with a complaint of passing thin stream of urine. One month earlier the child had been previously diagnosed with bilateral grade five Vesicoureteral reflux for which he had been surgically treated by bilateral ureter reimplantation. Following this, a complaint of UTI was reported and Escherichia coli was isolated. The patient on current admission had meatal stenosis with a complaint of poor stream of urine. The child was immunized as per the Indian primary immunization schedule. On Physical examination the child had a pulse rate of 90 per minute and Blood pressure 110/80 $\mathrm{mmHg}$. Hematological findings showed 10\% Monocyte, 1\% Basophils, $51 \%$ Neutrophils, $38 \%$ Lymphocytes. Hemoglobin was $10.8 \mathrm{mg} / \mathrm{dl}$, Platelet count was $465 \times 10^{3}$ per microliter and Total WBCs was found to be $20.1 \times 10^{3}$ per microliter. Urine specimen was collected after receiving consent from parents. Specimen was sent to laboratory for further investigations. Biochemical analysis of urine revealed that the $\mathrm{pH}$ was 6.5 with the color being pale yellow and specific gravity being $<1.005$. Traces of protein were present with sugar being absent in the urine. Blood urine was +1 ( 25 erythro- 
cyte/microliter) and urine leukocyte was +3 ( $\geq 500$ leukocyte/ml). Mid-stream urine was sent to microbiology laboratory and processed as per standard operating procedure. Macroscopic appearance of urine was turbid. Direct microscopy of urine specimen stained with gram stain showed numerous pus cells with gram negative bacilli. Semi-quantitative culture on blood agar yielded small colonies with colony count of $>10^{5} \mathrm{CFU} / \mathrm{mlafter}$ incubated at $37^{\circ} \mathrm{C}$ for 24 hours. MacConkey agar plate showed pinpoint lactose fermenting colonies. On further incubation at $37^{\circ} \mathrm{C}$ for 72 hours the size of did not change (Figure 1 and 2). Isolated colonies were picked up for identification and phenotypic characterization. Motility was confirmed with hanging drop method, isolates were non-motile. It was Gram negative bacilli. Delayed Catalase reaction and oxidase reaction was negative. Glucose was fermented with acid and gas. Indole, Methyl red and nitrate reduction test was positive. Sugars like mannitol, lactose and xylose were fermented. It failed to ferment sucrose and sorbitol. Lysine was not decarboxylated. Colonies were suspected to be Small colony variant Escherichia coli. Further identification was confirmed by using automated identification system (Vitek-2 compact, Biomerieux, France). The result obtained was Escherichia coli with the similarity of $99 \%$.Antimicrobial Susceptibility testing was performed by Kirby - Bauer disk diffusion method according to CLSI guidelines and the isolate was identified as Small colony variant Escherichia coli that did not grow on Mueller Hinton agar, so it was tested on Mueller Hinton agar supplemented with 5\% human blood. It was resistant to Cefuroxime $(30 \mu \mathrm{g})$, Norfloxacin $(10 \mu \mathrm{g})$, Ampicillin $(10 \mu \mathrm{g})$, Ceftriaxone $(30 \mu \mathrm{g})$, Nitilmicin $(30 \mu \mathrm{g})$, Amoxicillin Calvulinic acid $(20-10 \mu \mathrm{g})$, amikacin $(30 \mu \mathrm{g})$, Aztreonam $(30 \mu \mathrm{g})$, Gentamicin $(10 \mu \mathrm{g})$, Cefepime $(30 \mu \mathrm{g})$ and sensitive to Imipenem $(10 \mu \mathrm{g})$, Cotrimoxazole $(1.25-23.75 \mu \mathrm{g})$, Piperacillin Tazobactam (100-10 $\mu \mathrm{g})$ and Cefoperazone - Sulbactam (75-30 $\mu \mathrm{g})$. This isolate was identified as ESBL producer. Repeated urine culture and sensitivity yielded the same results.

\section{Discussion}

To our knowledge, this is a first report of recurrent urinary tract infection caused by small colony variant of Escherichia coli with ESBL producer after surgical treatment of bilateral grade five vesicouteralreflex. There are only few case reports of small colony variant of Escherichia coli in the literature. The repeated admission of the patient with the laboratory results indicates that the present case was that of recurrent urinary tract infection. The infection caused earlier was by Escherichia coli sensitive to the aminoglycosides and beta-lactam antibiotics that are used in treatment of urinary tract infections. However the bacteria isolated later in the subsequent admission was not the same as the previous isolate. It turned out to be ESBL producer and also showed a change in the colony characteristics and biochemical tests results showed catalase weak positive and failed to ferment sorbitol. The bacteria did not grow in the routine culture media in 24 hours and took a longer time to grow at $37^{\circ} \mathrm{C}$. In this case, the patient was treated with carbapenems during previous episode of UTI, unfortunately patient did not show recovery from the infection and currently progressed to recurrent UTI with Escherichia coli small colony variants, and probably indicating that due to the prolonged administration of the antibiotics given earlier due to surgery may have resulted in emergence of antibiotic stress induced small colony variants. In case of samples sent from patients who received antibiotic treatment for a long period, clinical microbiology laboratories should be on alert of small colony variant, and proper methods should be used to identify these pathogens. When there is an infection resistant to treatment, persistent or not responding to proper antimicrobial therapy small colony variant of Escherichia coli should be considered. In these situations, clinicians should ask the clinical microbiology laboratory to carefully look into identification of small colony variants, which might have an impact on the selection of treatment regime as small colony variants tend to resist intracellular killing and are more resistant to antibiotics.

\section{References}

[1] B Foxman (March 1990). Recurring urinary tract infection: incidence and risk factors. A.M J Public Health 80(3), 331-333 http://dx.doi.org/10.2105/AJPH.80.3.331.

[2] Charlotte A. Colwell (Oct 1946). Small Colony Variants of Escherichia coli Small Colony Variants of Escherichia coli Small Colony Variants of Escherichia Coli. J Bacteriol 52(4), 417-422.

[3] Hooton TM \& Stamm WE (Sep 1997). Diagnosis and treatment of uncomplicated urinary tract infection. Infect Dis Clin North Am. 11(3), 551-81 http://dx.doi.org/10.1016/S0891-5520 (05)70373-1.

[4] Harald Seifert, Hilmar Wisplingh off, Petra Schnabel, Christ of von Eiff (Oct 2003). Small Colony Variants of Staphylococcus aureus and Pacemaker-related Infection. Emerg Infect Dis 9(10), 1316-1318 http://dx.doi.org/10.3201/eid0910.0302000.

[5] L J Mandel, E Murphy, N H Steigbigel, M H Miller (Oct 1998). Gentamicin uptake in Staphylococcus aureus possessing plasmid-encoded, aminoglycoside-modifying enzymes. Antimicrob Agents Chemother 26(4), 563-569. http://dx.doi.org/10.1128/AAC.26.4.563.

[6] Mulvey MA, Lopez-Boado YS, Wilson CL, Roth R, Parks WC, Heuser J, Hultgren SJ (20 Nov 1998). Induction and evasion of host defenses by type 1-piliated uropathogenic Escherichia coli. Science. 282(5393), 1494-7. http://dx.doi.org/10.1126/science.282.5393.1494.

[7] Proctor RA, Van Langevelde P, Kristjansson M, Maslow JN, Arbeit RD (Jan 1995). Persistent and relapsing infections associated with small-colony variants of Staphylococcus aureus. Clin Infect Dis. 20(1) 95-102. http://dx.doi.org/10.1093/clinids/20.1.95.

[8] Proctor RA, von Eiff C, Kahl BC, Becker K, McNamara P, Herrmann M, Peters G (Apr 2006).Small colony variants:a pathogenic form of bacteria that facilitates persistent and recurrent infections. Nat Rev Microbiol. 4(4), 295-305. http://dx.doi.org/10.1038/nrmicro1384.

[9] Roggenkamp A, Sing A, Hornef M, Brunner U, Autenrieth IB, Heesemann J (Sep 1998). Chronic prosthetic hip infection caused by a small-colony variant of Escherichia coli. J Clin Microbiol. 36(9), 2530-4.

[10] Sabeen Habib (July 2012). Highlights for Management of a Child with a Urinary Tract Infection. Int J Pediatr. doi: 10.1155/2012/943653. http://dx.doi.org/10.1155/2012/943653.

[11] Vaudaux P, Kelley WL, Lew DP (Oct 2006). Staphylococcus aureus small colony variants: difficult to diagnose and difficult to treat. Clin Infect Dis. 15; 43(8), 968-70. 